J. Clin. Chem. Clin. Biochem.

Vol. 15, 1977, pp. 629-634

\title{
Ein einfaches Verfahren zur radioimmunologischen Bestimmung von Sexualsteroiden im Plasma
}

\author{
Von G. Ittrich
}

Bereich Medizin (Charité) der Humboldt-Universität zu Berlin, Frauenklinik (Direktor: Prof. Dr. med. habil. H. Bayer) Abteilung für gynäkologische Labordiagnostik

(Eingegangen am 9. Februar/1. August 1977)

\section{Meiner Frau Sabine gewidmet}

Zusammenfassung: Es wird ein einfaches Verfahren zur radioimmunologischen Bestimmung von Plasmasteroiden beschrieben. Wesentliche Arbeitsschritte sind: Lösung von Extraktionsrückständen in $20 \mu \mathrm{l}$ Ethanol oder direkter Einsatz von $20 \mu$ Ethanolextrakt, 40 min Inkubation mit je $500 \mu$ Tracerlösung bekannter Tracermasse und Antiserumverdünnung bzw. Gelatinephosphatpuffer sowie Abtrennung der freien Radioaktivität durch kohlebeschichtetes Florisil. Die Berechnung der Werte erfolgt nach einer einfachen Gleichung unter Verwendung einer der Tracermasse äquivalenten Steroidmasse als Bezugsgröße.

\section{A simple method for the radioimmunologic determination of sex steroids in plasma}

Summary: A simple method for the determination of plasma steroids by radioimmunoassay is described. Essential steps are: Dissolution of extraction residues in $20 \mu \mathrm{l}$ ethyl alcohol or direct addition of $20 \mu \mathrm{l}$ alcoholic plasma extract to the incubation tubes, addition of tracer solution with known tracer mass and antiserum dilution or gelatine phosphate buffer, incubation for $\mathbf{4 0} \mathrm{min}$ and separation of free from bound radioactivity by charcoalcoated florisil. Plasma steroid levels are calculated by a simple equation, an immunologic equivalent to the tracer mass serves as the reference value.

\section{Einführung}

Radioimmunologische Verfahren zeigen bei bestechend einfacher Versuchsführung, einer durch die Immunreaktion bedingten relativ hohen Spezifität der Endpunktsbestimmung, großer Empfindlichkeit und guter Mechanisierbarkeit eine Reihe von Nachteilen. Neben der Abhängigkeit der Verfahren von der Qualität der Antiseren zählt hierzu besonders die Notwendigkeit zum Mitführen einer größeren $Z$ ahl von Referenzproben für die Anfertigung einer Standardkurve bei jedem Ansatz, so daß einzelne Plasmaproben rationell nicht bestimmt werden können. Häufig sind relativ lange Inkubationszeiten besonders bei lipidhalltigen Plasmaextrakten erforderlich, um eine vollständige Diffusion der Steroide durch die am Glas haftende Lipidschicht oder eine vollständige Lösung der Steroide in dem wäßrigen Reaktionsgemisch zu garantieren, und es gibt Probleme bei der Abtrennung des nicht gebundenen Tracers. So zeigen streufähige Adsorptionsmittel wie z.B. Florisịl bei guter Dosierbarkeit eine stark chargenabhängige Adsorption bei hoher Restaktivität; bessere Adsorptionseigenschaften finden sich bei Amberlitstreifen. Hier ist jedoch eine lange Adsorptionszeit bei „über-KopfRotation " in speziellen, verschraubbaren Reaktionsgefäßen erforderlich und die Zugabe der Streifen ist weniger gut mechanisierbar; bei ausgezeichneten Adsorptionseigenschaften sind Dextran-Kohle-Suspensionen, denen in zahlreichen Verfahren der Vorzug gegeben wird, mit automatischen Dosiergeräten nicht gleichmäßig dosierbar, die Suspension ist nicht sehr stabil, die Adsorptionszeit ist nicht exakt steuerbar, der Pipettierfehler wird vergrößert, und das Reaktionsgemisch muß anschließend zentrifugiert werden. Letzteres gilt auch für Fällungsverfahren z.B. mit Ammoniumsulfatlösung.

Die an sich sehr elegante Trennung der freien von der gebundenen Aktivität durch Einsatz polymerisierter, an Kunststoff gebundener oder als festhaftende Schicht auf die Wand der Inkubationsgefäße aufgebrachter Antikörper bringt neben technischen Problemen infolge der zusätzlich erforderlichen Behandlung des empfindlichen Antiserums neue Unsicherheitsfaktoren in die an sich schon recht störanfälligen immunologischen Systeme. Will man schließlich bei den bekannten Verfahren eine graphische Auswertung vermeiden, so wird ein hoher 
Aufwand für die Lineartransformation der Standardkurven und die Ermittlung der Zielgrößen durch einen Rechner erforderlich (1).

In dieser Mitteilung wird versucht, ungeachtet der prinzipiellen Fehlermöglichkeiten radioimmunologischer Bestimmungen in ungereinigten Extrakten, die genannten Nachteile unter besonderer Berücksichtigung der Praktikabilität zu beseitigen.

Da die Prinzipien radioimmunologischer Verfahren als bekannt vorausgesetzt werden können und wesentliche Punkte des nachstehend beschriebenen Verfahrens auch auf andere Methoden zur Wirkstoffbestimmung durch Sättigungsanalyse anwendbar sind, wird auf eine detaillierte Darstellung der immunologischen Parameter der hier verwendeten Antiseren und der davon abhängigen analytischen Kriterien verzichtet.

\section{Material und Methoden}

1. $\left[1,2,6,7-{ }^{3} \mathrm{H}\right]$ Progesteron, spezifische Aktivität: $3,44 \mathrm{TBq} /$ $\mathrm{mmol}$

$\left[1,2-{ }^{3} \mathrm{H}\right]$ Testosteron, spezifische Aktivität: $1,63 \mathrm{TBq} / \mathrm{mmol}$

$\left[6,7-{ }^{3} \mathrm{H}\right]$ Östradiol- $17 \beta$, spezifische Aktivität: $1,78 \mathrm{TBq} / \mathrm{mmol}$ (Hersteller: New England Nuclear Corp.)

[125 J]Testosteron-3-carboxymethyloxim-monojodtyramid, spezifische Aktivität (Ausgangsaktivität):

Präp. a) $27,29 \mathrm{TBq} / \mathrm{mmol}$

b) $50,88 \mathrm{TBq} / \mathrm{mmol}$

(Hersteller: Akademie der Wissenschaften der DDR, Zentralinstitut für Isotopen- und Strahlenforschung, Bereich SN)

2. Phosphatpuffer $\mathrm{pH} 7,0,1 \mathrm{~mol} / \mathrm{l} ; 0,154 \mathrm{~mol} / 1 \mathrm{NaCl}$; $0,0154 \mathrm{~mol} / \mathrm{I} \mathrm{NaN}_{3}$

3. Gelatine-Phosphatpuffer: $1 \mathrm{~g} / 1$ Gelatine in Phosphatpuffer

4. Tracerlösungen in Gelatine-Phosphatpuffer (etwa $0,6 \mathrm{MBq} / 1$ bei ${ }^{3} \mathrm{H}$-Markierung; etwa $1,3 \mathrm{MBq} / \mathrm{l}$ bei ${ }^{125} \mathrm{~J}$-Markierung) wurden, z.T. durch Zusatz von nichtmarkiertem Steroid, auf Konzentrationen von etwa $0,19 \mathrm{nmol} / 1$ (Progesteron)

$1,0 \mathrm{nmol} / 1$ (Östradiol-17 $\beta$ )

$1,4 \mathrm{nmol} / \mathrm{l}$ (Testosteron, tritiummarkiert)

$1,6 \mathrm{nmol} / \mathrm{l}\left(\right.$ (125-Jodmarkierung, $^{125}$ immunologisches Testosteron-

eingestellt.

Traceräquivalent)

5. Antiserumverdünnungen (Kaninchen) in Gelatine-Phosphatpuffer:

a) Anti-17 $\beta$-Östradiol-6-carboxymethyloxim-Rinderserumalbumin $1: 15000$,

b) Anti-Testosteron-3-carboxymethyloxim-Rinderserumalbumin $1: 25000$,

c) Anti-11-Hydroxy-progesteron-11-hemisuccinat-Rinderserumalbumin 1:5000.

Die Antiseren zeigten folgende Kreuzreaktionen (2):

a) Östradiol-17 $\beta=100 \%$ : Östron 4,3\%; Östriol 0,4\%; EthinylÖstradiol 5\%; Östradiol-3-methyläther, $16 \alpha$-Hydroxyöstron sowie 2-Methoxyöstron 1\%, Dehydroepiandrosteron, $17 \alpha$ Hydroxyprogesteron, $17 \beta$-Hydroxyprogesteron, Progesteron, Testosteron sowie Dihydrotestosteron $<0,1 \%$.

b) vgl. 1.c (3).

c) Progesteron $=100 \%$ : $11 \alpha$-Hydroxyprogesteron $46 \%$; $17 \alpha$ Hydroxyprogesteron 0,5\%; Cortisosteron 1,5\%; Androstendion 0,3\%, Pregnenolon 0,3\%; Pregnandiol $<0,1 \%$.

Die Antiserumverdünnungen wurden so eingestellt, daßß bei Zugabe zu dem gleichen Volumen an Tracerlösung 40 bis $60 \%$ des Tracers bei der weiter unten angegebenen Versuchsanordnung gebunden wurden.
6. Florisil, kohlebeschichtet: Florisil wurde getrocknet, mit $500 \mathrm{~g} / 1$ Saccharoselösung getränkt, getrocknet und verkohlt. Das Präparat wurde mehrfach in Wässer aufgeschwemmt, dabei wurden die feinen Partikel abdekantiert. Anschließend wurde dreimal mit Ethanol nachgewaschen, abgesaugt und getrocknet (Maschengröße 60 ... 100).

7. Szintillationslösung: $4 \mathrm{~g} \mathrm{PPO}^{1}$ ), 0,04 g POPOP 2 ), $10 \mathrm{ml}$ Ethanol mit Toluol auf $1000 \mathrm{ml}$ aufgefillt.

8. Eppendorf-Mikrolitersystem, verstellbarer Eppendorf-Pulverdosierer (Einhandbedienung, $1 \mathrm{~s}$ pro Dosierung, Meßgenauigkeit ca $\pm 1,5 \%$ ), Eppendorf-Pipette „4700“ (mit Spitzenauswerfer), Mikroliterspritze System ,Stützerbach“, gasdicht, $1 \ldots 50 \mu 1$

Arbeitsweise zur radioimmunologischen Bestimmung von Plasmasteroiden $(4,5,6)$

\section{Extraktdärstellung:}

Entsprechend der żu erwartenden Konzentration werden 50 bis $500 \mu \mathrm{l}$ Plasma mit $5 \mathrm{ml}$ Äther $10 \mathrm{~min}$ extrahiert, die wäßrige Phase wird durch Eintauchen in eine Trockeneis-AlkoholMischung oder in der Tiefkühltruhe eingefroren und der Ätherextrakt wird quantitativ in ein $10 \mathrm{ml}-$ Zentrifugenglas mit rundem Boden abgegossen.

Der Äther wird dann bei etwa $35^{\circ} \mathrm{C}$ im gefilterten L uftstrom vertrieben (Zusatz von etwa $0,3 \mathrm{ml}$ Benzol nach kurzzeitigem Abstellen des Luftstroms kurz vor Ende der Destillation, um Wasserspuren zu vertreiben und den Extrakt am Boden zu konzentrieren). Der Rückstand wird mit $20 \mu \mathrm{l}$ Ethanol gut benetzt. Bei hohen Steroidkonzentrationen werden 50 bis $200 \mu \mathrm{l}$ Plasma mit 1 bis $0,8 \mathrm{ml}$ Ethanol $10 \mathrm{~min}$ geschüttelt, und nach Zentrifugieren werden $20 \mu$ Ü Überstand direkt zur Immunreaktion eingesetzt ( $\mathbf{B}_{\mathbf{x}}$-Proben; Doppelbestimmungen). Da bei Ethanolfällung auch Steroidkonjugate extrahiert werden, können bei Verwendung von wenig speżifischen Antiseren Differenzen zu den bei Ätherextraktion erhaltenen Werten auftreten.

\section{Meßansatz für die Immunreaktion}

Je 3 Gläschen zur Bestimmung der Restaktivität ( $R_{0}$-Proben) und zur Bestimmung der gebundenen Aktivität ( $B_{0}-$ Proben) werden mit $20 \mu \mathrm{l}$ Ethanol versetzt. Gleichzeitig werden für Kontrollmessungen Standardsubstanzen mit angesetzt $\left(B_{\text {ref }}\right.$ Proben).

Anschließend gibt man in alle Gläschen $500 \mu 1$ Tracerlösung, mischt kurz durch Umschwenken, versetzt die $\mathbf{R}_{0}$-Proben mit $500 \mu$ l Gelatine-Phosphatpuffer und die übrigen Proben mit $500 \mu$ l Antiserumverdünnung, mischt wieder kurz durch Umschwenken und läßt den Ansatz $20 \mathrm{~min}$ bei etwa $20^{\circ} \mathrm{C}$ sowie $20 \mathrm{~min}$ im Eisbad stehen. Dabei wird gelegentlich leicht geschwenkt. Schließlich erfolgt zügig ein Zusatz von $40 \ldots 60 \mathrm{mg}$ kohlebeschichtetem Florisil (möglichst genau dosieren), der Ansatz wird $15 \mathrm{~min}$ mit einem Vibrator kräftig gerüttelt, um das Adsorptionsmittel gut aufzuwirbeln, man läßt etwa $15 \mathrm{~s}$ absetzen und entnimmt exakt $500 \ldots 700 \mu$ Überstand zur Messung der Radioaktivität.

- Bei tritiummarkierten Steroiden wird das Aliquot in den Zählküvetten durch leichtes Schütteln für etwa $30 \mathrm{~min}$ mit $5 \mathrm{ml}$ Szintillationslösung extrahiert.

- Die Berechnung erfolgt nach

$$
\text { I. } \quad p_{x}=\left(\frac{B_{0}-R}{B_{x}-R}-1\right) \cdot p_{50} \cdot \text { Verdünnungsfaktor }
$$

Bei der Messung von Ätherextrakten wird ein entsprechender Ätherleerwert vom Endwert abgezogen.

Der lineare Meßbereich für die jeweils verwendeten Systeme muß zunächst durch Herstellung von Standardkurven $\left(x^{\prime}=p_{\text {ref }}\right.$; $\left.y^{\prime}=\frac{B_{0}-R}{B_{\text {ref }}-R}-1\right)$ bestimmt werden.

Der gewünschte Meßbereich wird dabei durch Ëinsetzen einer geeigneten Tracermasse vorgewählt, die gegebenenfalls durch

\footnotetext{
1) 2,5-Diphenyloxazol

2) 1,4: bis -2-(5-phenyloxazolyl)benzol
} 
Zusatz von nichtmarkiertem Steroid entsprechend eingestellt werden kann. Die der - von der Herstellung der Tracerlösung und der spezifischen Aktivität her bekannten - Tracermasse immunologisch äquivalente Masse an nichtmarkiertem Steroid $p_{50}$ ergibt sich aus der Steigung des linearen Teils der Standardkurve $\left(p_{50}=\cot \alpha\right)$. Ist für ein bestimmtes System der lineare Bereich schon bekannt, so kann man sich - z.B. bei Wechsel der Tracercharge - auf wenige Meßpunkte (Mehrfachbestimmungen) beschränken, um den Wert von $\mathrm{p}_{50}$ ausreichend genau zu bestimmen. Bei den laufenden Serien wird dieser Wert dann durch Kontrollmessungen (Dreifachbestimmungen) von Standardsubstanzen ( $p_{\text {ref }}=\mathrm{ca} .0,8$ und $1,5 \cdot \mathrm{p}_{50}$ ) überprüft. Die Auswertung erfolgt entweder graphisch oder durch Berechnung nach

$$
\text { II. } \quad p_{r e f}=\left(\frac{B_{0}-R}{B_{\text {ref }}-R}-1\right) \cdot p_{50} \text {. }
$$

Es empfiehlt sich, entsprechend dem Probendurchgang des Labors die Arbeitslösungen in größeren Mengen anzusetzen und diese in kleineren Portionen (2-4 Ansätze) bei $-20^{\circ} \mathrm{C}$ aufzubewahren. Nach den bisherigen Erfahrungen ist es zumindest zweckmäßig, von den genuinen, in $1 \mathrm{ml}$-Portionen eingefrorenen Antiseren nach dem Auftauen sogleich $1: 10$-Verdünnungen und von letzteren $(1 \mathrm{ml})$ ebenfalls sogleich $1: 100$-Verdünnungen mit Phosphatpuffer herzustellen und alle Verdünnungen nach Herstellung der Arbeitslösung in $1 \mathrm{ml}$-Portionen sofort wieder einzufrieren. Auf diese Weise braucht nur jeweils eine der letzten Verdünnungen aufgetaut zu werden und die Qualität des Antiserums bleibt über lange Zeit konstant. Als Stammlösung zur Herstellung der Tracerarbeitslösung wurden methanolische Lösungen verwendet, die eine für einen Ansatz ausreichende Tracermasse pro $\mathrm{ml}$ enthielten und ebenfalls bei $-20^{\circ} \mathrm{C}$ aufbewahrt wurden.

\section{Bezugszeichen}

$\mathrm{p}_{\mathbf{x}} \quad=$ gesuchte Steroidmasse

pref. = bekannte Steroidmasse im Reaktionsgemisch

p50 = die der Tracermasse immunologisch äquivalente Masse an nichtmarkiertem Steroid, mit der eine 50\%ige Isotopenverdünnung bzw. Verdrängung des Tracers aus der Bindung am Antikörper erzielt wird

$p_{\text {tracer }}=$ Tracermasse im Reaktionsgemisch

$\mathrm{p}_{\mathrm{as}}=$ am Antikörper gebundene Tracermasse

$\mathrm{B}_{\mathrm{O}} \quad=$ Impulsrate der Proben ohne Zusatz von nichtmarkiertem Steroid, Mittelwert, $0,5 \mathrm{ml}$ Reaktionsgemisch

$B_{\text {ref }}=$ Impulsrate der Proben mit Zusatz einer bekannten Menge an nichtmarkiertem Steroid, $0,5 \mathrm{ml}$ Reaktionsgemisch

$\mathbf{B}_{\mathbf{x}} \quad=$ Impulsrate der unbekannten Probe, 0,5 $\mathrm{ml}$ Reaktionsgemisch

$R_{0} \quad=$ Impulsrate der Proben ohne Zusatz von Antiserumverdünnung, Restaktivität (Mittelwert), 0,5 ml Reaktions-

$\mathbf{R}=\begin{aligned} & \text { gemisch } \\ & \text { korrigierte Restaktivität }(\end{aligned} \mathbf{R}_{\mathbf{0}}\left(1-\frac{\mathbf{B}_{0}-\mathbf{R}_{\mathbf{0}}}{\overline{\overline{\mathrm{T}}}-\mathbf{R}_{\mathbf{0}}}\right)$

$\mathrm{T}=$ Gesamtimpulsrate in 0,25 ml Tracer-Arbeitslösung bzw. in $0,5 \mathrm{ml}$ Reaktionsgemisch.

\section{Ergebnisse}

Korrelation $z$ wischen berechneten und eingesetzten Werten

Bei Messung bekannter Mengen an Steroiden und Berechnung nach Gleichung II aus den durch vorhergehende Bestimmung bekannten $\mathrm{p}_{\mathbf{5 0}}$-Werten fanden sich die in Tabelle 1 dargestellten Ergebnisse. Die in der Tabelle dargestellten Werte können auch als Punkte entsprechender Standardkurven aufgefaßt werden $(x=$ eingesetztes

Steroid, $y=\frac{\text { gefundener Wert }}{\mathrm{p}_{\text {so }}}$

\section{Variationskoeffizient bei Bestimmungen im Plasma}

Bei Berechnung des VK aus jeweils 20 Doppelbestimmungen im Plasma ( $\ddot{A}$ therextraktion) ergaben sich für Östradiol-17 $\beta$ und Testosteron Werte von $6,5 \%$ bzw. $6,3 \%$ im Bereich von 0,5 bis $2 \cdot \mathrm{p}_{50}$ (etwa 60 bis $230 \mathrm{pg}$ bzw. ca. 120 bis 470 pg pro Probe). Für Progesteron ergab sich ein VK von 5,6\% im Bereich zwischen 1 und 3. $\mathrm{p}_{50}$ ( 30 bis $90 \mathrm{pg}$ pro Probe). Wurden in dem genannten Bereich gleiche Plasmaproben in unterschiedlichen Ansätzen wiederholt gemessen, so ergab sich eine Abweichung der aus Doppelbestimmungen ermittelten Mittelwerte von $<7 \%$ (Östradiol- und Testosteronbestimmungen, jeweils 4 Plasmaproben, 5 unterschiedliche Ansätze).

\section{Empfindlichkeit}

Der Ätherleerwert betrug $0,015 \pm 0,005 \cdot \mathrm{p}_{50}(1,6$ $\pm 0,6 \mathrm{pg}$; Östradiolbestimmung).

Werte um $0,1 \cdot \mathrm{p}_{50}(\geqq 2 \mathrm{SD})$ können noch gemessen werden (etwa 10 pg Östradiol; 20 pg Testosteron; 5 pg Progesteron).

\section{Wiederauffindung}

Bei Zusatz von 30 bis $200 \mathrm{pg}{ }^{3} \mathrm{H}$-markierter Steroide $\mathrm{zu}$ $200 \mu$ Plasma ergaben sich Wiederfindungsraten von $98 \pm 3 \%$ (Progesteron und Testosteron) bzw. $97 \pm 3 \%$ (Östradiol) bei einmaliger Extraktion mit $5 \mathrm{ml}$ Ethyläther.

Bei Alkoholfällung (0,05 ml Plasma, $1 \mathrm{ml}$ Ethanol; Messung in $20 \mu \mathrm{l}$ Uberstand) errechneten sich entsprechende Werte zwischen 96 und 99\% (VK = 9\%).

Wiederauffindungsraten von $100 \mathrm{pg}$ Steroid bei Zusatz zu 0,2 ml Gelatine-Phosphatpuffer, Ätherextraktion und radioimmunologischer Bestimmung betrugen $98 \pm 7 \%$ (Östradiol), $97 \pm 8 \%$ (Testosteron) und $99 \pm 7 \%$ (Progesteron).

Tab. 1. Messung bekannter Steroidmengen und Berechnung der Werte nach Gleichung I.

\begin{tabular}{|c|c|c|c|}
\hline \multirow{2}{*}{$\begin{array}{l}\text { Eingesetzt } \\
\text { (pg) } \\
\text { Östradiol-17 } \\
10 \\
20 \\
50 \\
100 \\
200\end{array}$} & \multirow{2}{*}{$\begin{array}{l}\begin{array}{l}\text { P50 } \\
\text { (pg) }\end{array} \\
115\end{array}$} & \multicolumn{2}{|c|}{$\begin{array}{l}\text { Gefunden (Mittelwerte aus } \\
\text { Doppelbestimmungen) (pg) }\end{array}$} \\
\hline & & $\begin{array}{c}\text { Nov. } 76 \\
9,7 \\
19,6 \\
45,3 \\
96,2 \\
200,1\end{array}$ & $\begin{array}{c}\text { Dez. } 76 \\
6,7 \\
19,5 \\
55,1 \\
96,0 \\
200,2\end{array}$ \\
\hline $\begin{array}{l}\text { Testosteron } \\
25 \\
50 \\
100 \\
200 \\
400\end{array}$ & 233 & $\begin{array}{r}23,4 \\
52,3 \\
94,5 \\
206,0 \\
399,0\end{array}$ & \\
\hline $\begin{array}{l}\text { Progesteron } \\
12,5 \\
25 \\
50 \\
100\end{array}$ & 29,5 & $\begin{array}{r}15,9 \\
25,6 \\
48,0 \\
100,1\end{array}$ & \\
\hline
\end{tabular}


Bei Zusatzversuchen in unterschiedlichen Patientenseren ( $100 \mathrm{pg} ; 0,2 \mathrm{ml}$ ) ergaben sich nach Abzug der „Leerwerte“ Wiederauffindungsraten von $110 \pm 6 \mathrm{pg}$ (Kinder), $115 \pm 7 \mathrm{pg}$ (Frauen, Proliferationsphase) bzw. $125 \pm 8$ pg (Männer) Östradiol. Über diese, die Spezifität des Antiserums betreffenden Abweichungen und detaillierte klinische Ergebnisse wird an anderer Stelle berichtet werden (6).

\section{Linearer Meßbereich:}

Bei den hier verwendeten Systemen lag der praktisch als linear anzusehende Meßbereich für Östradiol-17 $\beta$ zwischen 0 und $300 \mathrm{pg}\left(40 \%\right.$ Bindung, $\left.\mathrm{p}_{50}=115 \mathrm{pg}\right)$, für Testosteron zwischen 0 und $>400 \mathrm{pg}(68 \% \mathrm{Bin}$ dung, $\mathrm{p}_{50}=233 \mathrm{pg}$ ) und für Progesteron zwischen 0 und $100 \mathrm{pg}\left(62 \%\right.$ Bindung; $\left.\mathrm{p}_{50}=30 \mathrm{pg}\right)$.

\section{Konstanz der Bezugsgröße $\mathrm{p}_{\mathbf{5 0}}$ :}

Jeweils 10 Standardkurven (6 Meßpunkte in Doppelbestimmungen), die über einen Zeitraum von 6 Wochen mit jeweils frisch aus derselben Stammlösung $(1: 10$ Antiserumverdünnung, Tracer-Stammlösung; wiederholt gewechselte Adsorptionsmittelchargen) hergestellten Arbeitslösungen angefertigt worden waren, zeigten einen Variationskoeffizienten von $9,5 \%$ für $\mathrm{p}_{50}$ ( $\cot \alpha$, graphisch ermittelt).

Wurden in größerem Quantum hergestellte Arbeitslösungen in kleinen Portionen nach Art eines Testbestecks für jeden Ansatz eingefroren, so ergab sich bei Verwendung einer einzigen Adsorptionsmittelcharge unter vergleichbaren Bedingungen ein $\mathrm{VK}$ von $<1 \%$ für $\mathrm{p}_{50}$ (6 Kontrollen in Zehnfachbestimmungen, $\mathrm{p}_{50}$ berechnet).

Eine Auswertung von 16 über einen Zeitraum von 4 Monaten durchgeführten Ansätzen unter Bedingungen der klinischen Routinediagnostik (Ansatz mit vorverdünnten Stammlösungen, 0,1 bzw. $1 \mathrm{ml}$ je Ansatz, nur gelegentlich gewechselte Adsorptionsmittelchargen) ergab einen Variationskoeffizienten von $3,3 \%$ für $p_{50}$. Die beschriebenen Ergebnisse wurden in erster Linie bei Östradiolbestimmungen gefunden. Bei den in geringerer Zahl durchgeführten Testosteron- und Progesteronbestimmungen fanden sich etwa vergleichbare Werte.

\section{Inkubationszeit:}

Messungen der gebundenen Aktivität nach $20 \mathrm{~min}$ und $60 \mathrm{~min}$ Inkubationszeit bei $20^{\circ} \mathrm{C}$, gefolgt von $20 \mathrm{~min}$ Inkubation bei $0^{\circ} \mathrm{C}$ ergaben keine meßbaren Unterschiede (Östradiolbestimmung).

\section{Plasmawerte:}

Im Verlauf des Menstruationszyklus fanden sich bei Frauen in den bisher vorliegenden Untersuchungen Plasmawerte zwischen 0,18 und $1,8 \mathrm{nmol} / 1$ Östradiol, die nach Stimulierung der Ovarien mit gonadotropen Hormonen auf das Zehnfache des Ausgangswertes an- stiegen. Bei Mädchen vor der Menarche fanden sich Werte unter $0,15 \mathrm{nmol} / 1$ Östradiol.

Bisher bei Männern gefundene Testosteronwerte lagen bei 14,0 . . 35,0 nmol/1 Plasma, bei Frauen fanden sich Werte zwischen 1,4 und 3,0 nmol/l (Testosteron + Dihydrotestosteron) sowie Progesteronwerte zwischen $1,6 \mathrm{nmol} / 1$ (Proliferationsphase) und 25,4 nmol/1 (Sekretionsphase).

\section{Diskussion}

\section{Spezifität:}

Die Spezifität des hier beschriebenen Verfahrens beruht wie bei anderen analog aufgebauten Verfahren - abgesehen von einer geringen Selektivität der Ätherextraktion - allein auf der Spezifität der verwendeten Antiseren. Eine richtige Interpretation klinischer Ergebnisse setzt daher die Kenntnis über die Spezifität der jeweils verwendeten Antiseren und mögliche Kreuzreaktionen speziell in vergleichsweise niedrigen Konzentrationsbereichen und bei Anwesenheit unphysiologisch hoher Mengen immunreaktiver Metaboliten oder Pharmaka voraus. Daher sind auch hier wie in anderen Verfahren (7) von Fall zu Fall Praktikabilität und Spezifität gegeneinander abzuwägen und gegebenenfalls durch entsprechende Vortrennungen interferierende Verbindungen zu eliminieren. Ein Vergleịch der hier gefundenen Plasmawerte mit bekannten Werten deutet jedoch auf eine für bestimmte klinische Fragestellungen hịreichende Spezifität.

\section{Ethanolzusatz:}

Durch den Zusatz einer geringen, die Immunreaktion nicht wesentlich beeinflussenden Menge an Ethanol werden Steroidrückstände sofort in Lösung gebracht bzw. bei entsprechender Extraktdarstellung (8) als Lösung direkt in die Reaktion eingesetzt. Bei der nachfolgenden Zugabe der Tracerlösung erfolgt daher momentan die Isotopenverdünnung, so daß weder Lösungs- oder Diffusionsvorgänge noch die zur Verdrängung von bereits am Antiserum gebundenem Tracer erforderliche Zeit, sondern nur die Reaktionsgeschwindigkeit der Bindung des Steroid-Tracer-Gemisches am Antiserum als Zeitfaktor in Erscheinung treten kann. Diese Reaktion ist unter den gegebenen Bedingungen nach $20 \mathrm{~min}$ bei $20^{\circ} \mathrm{C}$ und $20 \mathrm{~min}$ Kühlung weitgehend abgeschlossen. Diese Verkürzung der Inkubätionszeit ist in bestimmten Fällen der klinischen Ưberwachung und Therapiekontrolle von Bedeutung.

\section{Abtrennung der freien Radioaktivität:}

Der Einsatz eines streufähigen, sich schnell absetzenden Adsorptionsmittels ermöglicht die Verwendung von Pulverdosierern. Bei der Eppendorf-Pulverpipette z.B. wird für die Zugabe von je $40 \pm .0,7 \mathrm{mg}$ Adsorptionsmittel in 50 Röhrchen bei Einhandbedienung weniger als $1 \mathrm{~min}$ benötigt. Die Adsorptionszeit läßt sich 
durch Festlegung der Vibrationszeit exakt steuern, bei großen Serien tritt kein Adsorptionszeitgefalle auf und das Adsorptionsmittel setzt sich schnell ab, sodaß die bei Dextran-Kohle-Suspensionen erforderliche Zentrifugation hier entfällt.

Da sich schon geringe Streuungen der Zählraten in der Zielgröße deutlich bemerkbar machen, wirkt sich der durch Fortfall einer zusätzlichen Pipettierung verringerte Volumenfehler vorteilhaft aus.

Die Restaktivität bei den hier verwendeten, im Laboransatz hergestellten Chargen kohlebeschichteten Florisils lag mit 2-4\% im Vergleich zu dextranbeschichteten Aktivkohlesuspensionen (1-2\%), Amberlitstreifen (5-10\% bei $1 \mathrm{~h}$ Adsorption) oder Florisil (10-15\%) in einem günstigen Bereich. Florisil wurde in erster Linie wegen seiner guten Streufähigkeit als anorganischer Träger gewählt; als ebenso geeignet und in seinen Adsorptionseigenschaften noch etwas selektiver (Restaktivität unter $1 \%$ ) erwies sich entsprechend beschichtetes streufähiges Kieselgel geeigneter Korngröße $(>40 \mu \mathrm{m})$.

\section{Auswertungsverfahren:}

Einschränkende Voraussetzung für das hier gewählte Auswertungsverfahren ist - im Gegensatz zu einem anderen Modell (9) - die Wahl eines Meßbereiches, in dem weitgehend die Bedingungen einer einfachen Isotopenverdünnung angenommen werden können, d.h. ein Bereich, in dem Anderungen der Dissoziation des Antikörper-Hapten-Komplexes oder der Zahl der Haftstellen infolge Erhöhung der Haptenkonzentration praktisch vernachlässigt werden können und in dem auch aus der gleichen Ursache resultierende Veränderungen der Restaktivität keinen wesentlichen Einfluß zeigen. Zwischen der systembedingt korrigierten Meßgröße $\left(y=\frac{B_{x}-R}{B_{0}-R}\right)$ und der im Reaktionsgemisch vorhandenen Steroidmasse, die sich aus der Tracermasse und der Masse an nichtmarkiertem Steroid zusammensetzt und żweckmäßigerweise auch auf einen gemeinsamen Nenner $\left(p_{50}\right)$ gebracht wird $\left(x=\frac{p_{\text {tracer }}}{p_{50}}+\frac{p_{x}}{p_{50}}\right.$ bzw., da $p_{\text {tracer }}$ und $\mathrm{p}_{50}$ als immunologisch äquivalent angesehen werden, $\left.x=\frac{p_{x}}{p_{50}}+1\right)$ kann daher - im Sinne einer einfachen Verdünnungskurve - die einfạche hyperbolische Beziehung $\mathrm{y} \rightleftharpoons \mathrm{x}^{-1}$ angenommen werden, die nach entsprechender Umformung die Gleichung

III. $\quad \frac{B_{0}-R}{B_{x}-R}-1=\frac{\hat{p}_{x}}{p_{50}}$ ergibt. Eine entsprechende weitere Umformung ergibt dann die zur Auswertung verwendete Gleichung I bazw. II.
Setzt man den linken Ausdruck in Gleichung III $=y^{\prime}$ und $\mathrm{p}_{\mathrm{x}}=\mathrm{x}^{\prime}$, so ergibt sich nach $\mathrm{y}^{\prime}=\operatorname{tg} \alpha \cdot \mathrm{x}^{\prime}$ der Wert $p_{50}$ aus der Steigung entsprechend angefertigter Standardkurven, die, definitionsgemäß von 0 beginnend, soweit linear sind, wie die anfangs erwähnte hyperbolische Beziehung gültig ist.

Umgekehrt werden entsprechende Standardkurven eine praktisch ausreichende Linearität vorausgesetzt allein durch den Wert $\mathrm{p}_{50}$ bestimmbar. Durch Vorwahl einer geeigneten Tracermasse können gleichzeitig wesentliche analytische Parameter, d.h. Meßbereich und Empfindlichkeit, im Prinzip sogar die Standardkurve, a priori aus der vom Hersteller angegebenen spezifischen Aktivität nach Bedarf eingestellt bzw. berechnet werden.

Wenn sich auch gelegentlich eine recht gute Übereinstimmung $\mathrm{zwischen}$ berechnetem und gefundenem Wert für $\mathrm{p}_{50}$ fand (Progesteron $100: 104 \%$ ) und auch bei den bisher verwendeten jodmarkierten Testosteronchargen eine unter Berücksichtigung immunologischer Differenzen zwischen Tracer und nichtmarkiertem Steroid befriedigende Übereinstimmung $\left(\mathrm{p}_{50}=130 \%\right.$ der berechneten Tracermasse) gefunden wurde, so ist es ungeachtet der Richtigkeit der Angaben des Herstellers dennoch unerläßlich, den - als eine für jedes System spezifische Konstante $z u$ betrachtenden-Wert $p_{50}$ durch Herstellung von Standardkurven primär möglichst genau zu bestimmen. Bei dem hier beschriebenen, offenbar recht stabilen System ergaben sich in zahlreichen Ansätzen nur geringe Abweichungen von diesem Wert. Daher konnte bei der vergleichsweise guten Präzision darauf verzichtet werden, bei jedem Ansatz den gesamten Meßbereich durch eine Vielzahl von Messungen zur Herstellung einer Standardkurve zu kontrollieren; es reicht nach den bisher in einigen tausend Messungen gemachten Erfahrungen aus, den Wert $p_{50}$ durch wenige Kontrollmessungen jeweils zu überprüfen, um Abweichungen zu erkennen und gegebenenfalls zu korrigieren, ohne die für die klinische Routinediagnostik geforderten Toleranzgrenzen zu überschreiten.

\section{Linearität der Standardkurven:}

Für die hier verwendeten Systeme wurden praktisch als linear anzusehende Bereiche $z w i s c h e n ~ 0$ und etwa $p_{x}=$ $8 \cdot p_{\text {as }}-p_{50}$, d. h. bei $50 \%$ Bindung eine Linearität bis etwa $75 \%$ Verdrängung, gefunden. Bei Verwendung derselben Antiseren wurden entsprechende Bereiche auch für Systeme mit Abtrennung der freien Aktivität durch Dextran-Kohle-Suspension oder Amberlitstreifen gefunden. Eine orientierende Auswertung von Standardkurvenwerten aus anderen Laboratorien ergab ähnliche Befunde, und auch bei radioimmunologischen Verfahren zur Bestimmung von Lutropin oder Follitropin fanden sich lineare Bereiche bis 65 bzw. $80 \%$ Verdrängung (BYK Mallinckrodt-Testbesteck). 
Eignung für die Praxis:

Neben den bekannten Eigenschaften radioimmunologischer Verfahren zeichnet sich die beschriebene Arbeitstechnik durch eine kurze Versuchsdauer besonders bei Einsatz alkoholischer Extrakte aus, und die Zahl an mitzuführenden Standardproben kann ohne wesentlichen Verlust an Exaktheit verringert werden. Daher wird es möglich, gelegentlich anfallende, zur schnellen klinischen Information dringend benötigte Einzelproben mit ausreichender Sicherheit nicht nur schnell, sondern auch relativ rationell zu bestimmen.

\section{Literatur}

1. Rodbard, D. \& Lewald, J. E. (1970), Computer analysis of radioligand assay and radioimmunoassay data; in: Karolinska Symposia (Diczfalusy, E. ed.) Steroid Assay by Proteị Binding, Stockholm, S. 79-98.

2. Stahl, F.: Persönl. Mitt.

3. Stahl, F. (1975), Endokrinologie 65, 288-292.

4. Ittrich, G. (1976), Verfahren zur Bestimmung von Wirkstoffkonzentrationen durch Sättigungsanalyse, DDR-Patentanmeldung 8. 12. 1976.

5. Ittrich, G., Endokrinologie, im Druck.

\section{Danksagung}

Für die Utberlasssung von Antiseren und ${ }^{3} \mathrm{H}$-markierten Steroiden bin ich Herrn Dr. Stahl (Inst. f. experimentelle Endokrinologie, Bereich Medizin der Humboldt-Universität Berlin) söwie Herrn Prof. Dr. Busch (Tierklinik f. Geburtshilfe und Fortpflanzungsstörungen, Sektion Tierproduktion der Humboldt-Universität Berlin) zu Dank verpflichtet.

Herrn Prof. Dr. Vormum und Herrn Dr. Herzmann (ZI für Isotopen- und Strahlenforschung, Akademie der Wissenschaften der DDR, Berlin-Buch) danke ich besonders für die Überlassung von ${ }^{125} \mathrm{~J}$-markierten Steroïden.

Besonderer Dank gebührt meiner Mitarbeiterin B. Schäfer für exakte technische Assistenz.

6. Ittrich, G. (1977), Einige Aspekte zur radioimmunologischen Bestimmung von Plasmahormonen; Vortrag vor der Gesellschaft für Gynäkologie und Geburtshilfè, Berlin, Zbl. Gynäk. in Vorber.

7. Kuss, E. \& Goebel, R. (1976), diese Z. 14, 549-556.

8. Farmer, R. W. \& Pierce, Ch. E. (1974), Clin. Chem. 20, $411=414$.

9. Seaton, B., Lusty, Jane \& Watson, J. (1976), J. Steroid Biochem. 7, 511-516.

Dr. rer. nat. habil. Gerd Ittrich

Bereich Medizin (Charité) der Humboldt-Universität zu Berlin

Frauenklinik, Abt. f. gynäkol. Labordiagnostik

Tucholskystraße 2

DDR-104 Berlin 